Pacific Journal of Mathematic 


\title{
PUISEUX SERIES FOR RESONANCES AT AN EMBEDDED EIGENVALUE
}

\author{
JAMES S. HOWLAND
}

Let $H(\kappa)=T+\kappa B^{*} A$ be a self-adjoint perturbation of the self-adjoint operator $T$, and suppose that $T$ has an eigenvalue $\lambda_{0}$ of finite multiplicity $m$ embedded in its continuous spectrum. If the operator

$$
Q(z)=A(T-z)^{-1} B^{*}
$$

is bounded and can be continued meromorphically across the axis at $\lambda_{0}$, the asymptotic spectral concentration of the family $H(\kappa)$ at $\lambda_{0}$ is determined by the poles of

$$
\kappa A(H(\kappa)-z)^{-1} B^{*}=I-[I+\kappa Q(z)]^{-1} .
$$

These "resonances" can be expanded in a series of fractional powers of $\kappa$, and therefore have a unitarily invariant significance for the family $H(\kappa)$. An example shows that nonanalytic series may indeed occur; however, if a resonance is an actual eigenvalue of $H(\kappa)$ for all sufficiently small real $\kappa$, its series is analytic. Because the resonances cannot lie on the first sheet when $\kappa$ is real, these series must have a special form. In the generic case, they yield, as the lowest order approximation to the imaginary parts of the resonances, the famous Fermi's Golden Rule. The case when $\lambda_{0}$ is embedded at a branch point of (1) is studied by means of a simple example.

To outline briefly, Puiseux expansions are obtained in $\S 1$, and their special form is noted (c.f. [15, Theorem 4.2]). In $\S 2$, a study of these series for perturbations which remove the degeneracy at $\lambda_{0}$ leads to Fermi's Golden Rule. The discussion of spectral concentration in $\S 3$ relies heavily on the arguments of [3], particularly on a grouping of the resonances into "clusters" which act asymptoticly as a single simple pole. The examples appear in $\S 4$. The appendix contains a technical result which simplifies not only Theorem 3.1 but also [3, Theorem 2.1] (c.f. [3, p. 156; Note (1)]). The results proved here were announced in [4].

Simon $[14,15]$ has recently discussed a similar problem for $N$ body Hamiltonians with dilatation analytic interactions. It is of particular interest that the Balslev-Combes technique which he employs reduces the problem to that of an isolated eigenvalue of a non-self-adjoint operator. This gives an interesting insight into the occurrence of Puiseux series, and suggests that, in the general case, resonance series can be viewed as perturbation series for an isolated 
eigenvalue of a suitable non-self-adjoint operator. Simon considers eigenvalues of arbitrary finite multiplicity, and not, as erroneously remarked in [4], only simple multiplicity.

Eigenvalues embedded at "thresholds" are not considered by Simon. Mathematically, a threshold may be variously described as (i) a branch point of an appropriate function, (ii) a point where the absolutely continuous part of $T$ changes multiplicity, or (sometimes) (iii) an end point of the spectrum of $T$. The unperturbed eigenvalue in the second example of $\S 4$ is a threshold in all three senses. A slightly revised Golden Rule is shown to apply to this case.

Let us conclude this introduction with an observation about the invariant significance of "resonances". It is tempting, at first glance, to call a point $\Lambda$ a resonance of the self-adjoint operator $H$ if the continuation of some matrix element $\left((H-\zeta)^{-1} f, f\right)$ across the spectrum of $H$ has a pole at $\Lambda$. However, this definition is worthless; for if $H$ is the multiplication

$$
H f(x)=x f(x)
$$$$
-\infty<x<\infty
$$

(which is essentially the general case in which continuation is possible), then given any point $\Lambda$ in the lower half-plane, there is a rational function $f(x)$ for which the continuation of

$$
\left((H-\zeta)^{-1} f, f\right)=\int(x-\zeta)^{-1}|f(x)|^{2} d x
$$

has a pole at $\Lambda$. The "resonances" considered by various authors are always something more than this-poles of an $S$-matrix [11], of an integral operator [13], or (as here) of an operator-valued function. Accordingly, the definition of "resonance" is referred to some structure in addition to the operator $H$-such as outgoing subspaces, the representation of $H$ as a differential operator, or a decomposition $H=T+A B^{*}$.

While something of this sort is necessary in general, in the case of an analytic perturbation $H(\kappa)$ of an embedded eigenvalue, a unitarily invariant significance can be attached to a Puiseux series $\Lambda(\kappa)$ of "resonances" in the weak sense which we have scorned above. There is of course additional structure here, too: the analyticity of the families $H(\kappa)$ and $\Lambda(\kappa)$.

To be precise, suppose that $H(\kappa)$ is an analytic family [6, Chapter VII] of closed operators, self-adjoint for real $\kappa$, with essential spectrum independent of $\kappa$. Let $\lambda_{0}$ be an eigenvalue of $H(0)$ and assume that for some vector $f$

$$
\left((H(\kappa)-\zeta)^{-1} f, f\right)
$$


has a continuation $F(\zeta, \kappa)$ to a meromorphic function of $(\zeta, \kappa)$ for $|\kappa|<\delta$ and $\left|\zeta-\lambda_{0}\right|<\delta$. Assume further that

$$
\Lambda(\kappa)=\lambda_{0}+\beta \kappa^{n / p}+\cdots \quad \beta \neq 0
$$

is a pole of $F(\zeta, \kappa)$ for each $\kappa$. Since for small $\kappa$, the term $\beta \kappa^{n / p}$ dominates those which follow it, $\Lambda(\kappa)$ will be in the upper half-plane for $\kappa$ in certain sectors of the complex plane, and will therefore be an eigenvalue of $H(\kappa)$, because of the assumed invariance of the essential spectrum. Thus the same analytic family $\Lambda(\kappa)$ represents a "resonance" for some values of the perturbation parameter, and an actual eigenvalue of $H(\kappa)$ for others. Put differently, the resonances are continuations in $\kappa$ of eigenvalues of $H(\kappa)$, and have, therefore, a unitarily invariant significance for the family $H(\kappa)$.

1. Puiseux series. The following assumptions will be made throughout this article. For proofs of the various assertions, see [2, 7 , and 10].

Let $\mathscr{H}$ and $\mathscr{H}^{\prime}$ be separable Hilbert spaces. Let $T$ be a selfadjoint operator on $\mathscr{H}$ with resolvent $G(z)=(T-z)^{-1}$, and let $A$ and $B$ be closed, densely defined operators from $\mathscr{H}$ to $\mathscr{C}^{\prime}$ such that $\mathscr{D}(T) \subset \mathscr{D}(A) \cap \mathscr{D}(B)$ and

$$
(A x, B y)=(B x, A y) \text { for every } x, y \in \mathscr{D}(A) \cap \mathscr{D}(B) .
$$

Suppose that for every $z \in \rho(T)$, the operator $A G(z) B^{*}$, which is defined on $\mathscr{D}\left(B^{*}\right)$, has a bounded extension $Q(z)$ to $\mathscr{H}^{\prime}$, and that $I+Q(z)$ is invertible for some $z \in \rho(T)$. Then, for sufficiently small real $\kappa$, there is a self-adjoint extension $H(\kappa)$ of $T+\kappa B^{*} A$ the resolvent of which is

$$
R(z, \kappa)=G(z)-\kappa[B G(\bar{z})]^{*}[I+\kappa Q(z)]^{-1} A G(z)
$$

whenever $z \in \rho(T)$ and $I+\kappa Q(z)$ has a bounded inverse. In particular, $H(0)=T$ and $R(z, 0)=G(z)$. We shall write $H(\kappa)=\int \lambda d E_{\kappa}(\lambda)$. If $\mathscr{C}\left(A^{*}\right)$ denotes the smallest reducing subspace of $T$ which contains $\mathscr{R}\left(A^{*}\right)$, then $\mathscr{C}=\mathscr{M}\left(A^{*}\right) \cap \mathscr{M}\left(B^{*}\right)$ reduces both $H(\kappa)$ and $T$ and $H(\kappa)=T$ on $\mathscr{M}^{\perp}$. Only the parts of $H(\kappa)$ and $T$ in $\mathscr{C}$ are of interest in perturbation theory.

Let $\Omega$ be a neighborhood of a point $\lambda_{0}$ of the real axis, and $\Omega^{ \pm}=$ $\{z \in \Omega: \pm \operatorname{Im} z>0\}$. Assume that $Q(z)$ has a continuation $Q^{ \pm}(z)$ from $\Omega^{ \pm}$to $\Omega$, which is analytic on $\Omega$ except for a simple pole at $\lambda_{0}$ with residue of finite rank $m$. The part of $T$ in $\mathscr{M}$ is then absolutely continuous in $\Omega \cap \boldsymbol{R}$, except for an eigenvalue $\lambda_{0}$ of finite multiplicity equal to $m$. Since $Q^{+}(z)$ and $Q^{-}(z)$ do not in general agree on $\Omega$, 
the eigenvalue $\lambda_{0}$ is in general embedded in the absolutely continuous spectrum of $T$.

If we now write

$$
Q^{ \pm}(z)=Q_{c}^{ \pm}(z)+\left(\lambda_{0}-z\right)^{-1} F
$$

where $F$ has finite rank and $Q_{c}^{ \pm}(z)$ is analytic at $\lambda_{0}$, then $I+\kappa Q_{c}^{ \pm}(z)$ can be inverted by a Neumann series for $\left|z-\lambda_{0}\right|<\delta_{1}$ and $|\kappa|<\delta_{2}$ if $\delta_{1}$ and $\delta_{2}$ are sufficiently small. Hence, $A R(z, \kappa) B^{*}$ also has a bounded extension $Q_{1}(z, \kappa)$ for $\operatorname{Im} z \neq 0$, which has completely meromorphic (meromorphic with finite rank principal parts at all poles [2]) continuations $Q_{1}^{ \pm}(z, \kappa)$ from $\Omega^{ \pm}$to $\left|z-\lambda_{0}\right|<\delta_{1}$ satisfying

$$
\begin{aligned}
& I-\kappa Q_{1}^{ \pm}(z, \kappa)=\left[I+\kappa Q^{ \pm}(z)\right]^{-1} \\
& \quad=\left\{I+\kappa\left(\lambda_{0}-z\right)^{-1}\left[I+\kappa Q_{c}^{ \pm}(z)\right]^{-1} F\right\}^{-1}\left[I+\kappa Q_{c}^{ \pm}(z)\right]^{-1} .
\end{aligned}
$$

The poles of $Q_{1}^{ \pm}(z, \kappa)$ need not be real, but for real $\kappa$ do not lie in $\Omega^{ \pm}$; they are the resonances of this perturbation problem.

THEOREM 1.1. There is an analytic function $\Delta(z, \kappa)$ on a polydisc $\left\{(z, \kappa):\left|z-\lambda_{0}\right|<\delta_{1},|\kappa|<\hat{\delta}_{2}\right\}$ such that

(a) For $|\kappa|<\delta_{2}, \Delta(z, \kappa)$ has exactly $m$ zeros $z_{1}(\kappa), \cdots, z_{m}(\kappa)$ (repeated according to multiplicity) in $\left|z-\lambda_{0}\right|<\delta_{1}$, which are precisely the poles of $Q_{1}^{+}(z, \kappa)$ in $\left|z-\lambda_{0}\right|<\delta_{1}$. For $\kappa=0, z_{j}(0)=\lambda_{0}$ $(j=1, \cdots, m)$.

(b) If for some real $\kappa, z_{j}(\kappa)$ is real, then $z_{j}(\kappa)$ is an eigenvalue of $H(\kappa)$ of multiplicity equal to the multiplicity $m_{j}(\kappa)$ of $z_{j}(\kappa)$ as a zero of $\Delta(z, \kappa)$.

This result was proved in $[2, \S 5]$, except for analyticity of $\Delta(z, \kappa)$ which is clear from the construction of $\Delta(z, \kappa)$ (see equation (2.2) below). However, we have omitted the hypothesis of [2] that $Q(z)$ is compact. This can be done; for in [2] compactness was used only for two things: (a) to prove that $I+\kappa Q^{ \pm}(z)$ has a completely meromorphic inverse, and (b) to prove, by references to [10], that $H(\kappa)$ is self-adjoint for real $\kappa$. However, we have argued above that (a) holds here, while (b) holds for $\kappa$ sufficiently small [10, p. 59].

Note that [2] $F=A P_{0}\left[B P_{0}\right]^{*}$.

We shall now show that the resonances can be grouped into cycles, so that each of the $p$ elements of a cycle is one of the values of a series expansion in powers of $\kappa^{1 / p}$. Such series are known as Puiseux series [9, p. 130]. For their application to perturbation theory, see [6; Chapters II and VII].

THEOREM 1.2. The resonances $z_{1}(\kappa), \cdots, z_{m}(\kappa)$ may be labeled so 
that each $z_{j}(\kappa)$ has a Puiseux series expansion in $\kappa$. If

$$
z_{j}(\kappa)=\lambda_{0}+\alpha_{1} \omega^{j} \kappa^{1 / p}+\alpha_{2} \omega^{2 j} \kappa^{2 / p}+\cdots \quad(j=1, \cdots, p)
$$

is a given Puiseux cycle of resonances, where $\omega$ is a primitive pth root of unity, then either the series has the form

$$
z_{j}(\kappa)=\lambda_{0}+\alpha_{p} \kappa+\cdots+\alpha_{2 n p} \kappa^{2 n}+\alpha_{2 n p_{+1}} \omega^{j} \kappa^{2 n+1 / p}+\cdots
$$

where $\lambda_{0}, \alpha_{p}, \cdots, \alpha_{(2 n-1) p}$ are real and $\operatorname{Im} \alpha_{2 n p}<0$, or $p=1$ and all the coefficients $\alpha_{n}$ are real.

Moreover, the multiplicity $m_{j}(\kappa)$ is independent of $\kappa$ for $\kappa \neq 0$ and sufficiently small, and is the same for each element $z_{j}(\kappa)$ of a given Puiseux cycle.

In particular, if $z_{j}(\kappa)$ belongs to a Puiseux cycle with $p \geqq 2$, then $z_{j}(\kappa)$ is not real for all sufficiently small real $\kappa \neq 0$. Thus any actual embedded eigenvalues of $H(\kappa)$ are analytic.

COROLlary 1.3. For real $\kappa \neq 0$ sufficiently small, the multiplicity of point eigenvalues in the interval $\left(\lambda_{0}-\delta_{1}, \lambda_{0}+\delta_{1}\right)$ is independent of $\kappa$. If for some $j, z_{j}(\kappa)$ is real for all sufficiently small $\kappa$, then $z_{j}(\kappa)$ is analytic in $\kappa$.

Proof of Theorem 1.2. Since $\Delta(z, 0)=\left(\lambda_{0}-z\right)^{m}$, the Weierstrass Preparation Theorem [1, p. 188] yields that

$$
\Delta(z, \kappa)=\left[\left(z-\lambda_{0}\right)^{m}+g_{m-1}(\kappa)\left(z-\lambda_{0}\right)^{m-1}+\cdots+g_{0}(\kappa)\right] F(z, \kappa)
$$

where $g_{0}, \cdots, g_{m-1}$ and $F$ are analytic, $F\left(\lambda_{0}, 0\right) \neq 0$ and $g_{0}(0)=\cdots=$ $g_{m-1}(0)=0$. Thus $z_{1}(\kappa), \cdots, z_{m}(\kappa)$ are the zeros of a polynomial in $z$ with coefficients analytic in $\kappa$, namely $\Delta(z, \kappa) / F(z, \kappa)$. Hence, (c.f. [6, pp. 63-66]) $z_{1}(\kappa), \cdots, z_{m}(\kappa)$ are algebroidal functions having at most an algebraic singularity at $\kappa=0$, and must therefore have Puiseux series expansions. The statement about multiplicities is part of this theory.

Since $H(\kappa)$ is self-adjoint for real $\kappa, R(z, \kappa)$, and hence $Q_{1}^{+}(z, \kappa)$, is analytic for $\operatorname{Im} z>0$, so that in the cycle (1.4), one has $\operatorname{Im} z_{j}(\kappa) \leqq$ 0 for real $\kappa$, and each $j=1, \cdots, p$. Therefore, the first term of (1.4) with a nonreal coefficient must have negative imaginary part for all real $\kappa$ and $j=1, \cdots, p$. But this can only happen for an even integer power $\kappa^{2 n}$ where, moreover, $\operatorname{Im} \alpha_{2 n p}<0$. If all coefficients $\alpha_{n} \omega^{j n}$ are real, then because of the factor $\omega^{j n}$, we can only have $p=1$ or 2 . However, if $p=2$ and $\alpha_{n} \omega^{j n} \kappa^{n / 2}$ is the first nonzero term with $n$ odd, then changing $\kappa$ into $-\kappa$ introduces a factor $i$, so that by proper choice of $j$, the imaginary part of this term can be made positive. Since this cannot occur, we must have $p=1$. 
REMARK. With perhaps a mild additional hypothesis, stationary scattering theory [8] shows that, for real $\kappa$, the absolutely continuous parts of $H(\kappa)$ and $T$ in $\left(\lambda_{0}-\delta_{2}, \lambda_{0}+\delta_{2}\right)$ are unitarily equivalent.

2. Fermi's golden rule. In the simple case in which the perturbation $B^{*} A$ removes the degeneracy at $\lambda_{0}$, calculation of the resonances up to terms of order $\kappa^{2}$ leads to the venerable Golden Rule for the line widths $\Gamma_{j}(\kappa)$. In order to discuss this, we must recall the construction of $\Lambda(z, \kappa)[2, \S 5]$.

It was proved in [2, p. 329; Theorem 3.1] that the residue of $Q^{+}(z)$ at $\lambda_{0}$ is $-A P_{0}\left[B P_{0}\right]^{*}$, where $P_{0}$ is the orthogonal projection onto $\operatorname{ker}\left(T-\lambda_{0}\right)$. Hence the operator

$$
Q_{c}^{+}(z)=Q^{+}(z)-\left(\lambda_{0}-z\right)^{-1} A P_{0}\left[B P_{0}\right]^{*},
$$

which corresponds to the continuous part of $T$ near $\lambda_{0}$, is analytic on $\Omega$. According to [2, p. 335; Theorem 5.1]

$$
\Delta(z, \kappa)=\left(\lambda_{0}-z\right)^{m} \operatorname{det}\left[I+\left[I+\kappa Q_{c}^{+}(z)\right]^{-1} \kappa\left(\lambda_{0}-z\right)^{-1} A P_{0}\left[B P_{0}\right]^{*}\right] .
$$

Using the formula $\operatorname{det}(I+S T)=\operatorname{det}(I+T S)$ [6, p. 162; Problem 4.17] gives

$$
\Delta(z, \kappa)=\left(\lambda_{0}-z\right)^{m} \operatorname{det}\left\{I+\left[B P_{0}\right]^{*}\left[I+\kappa Q_{c}^{+}(z)\right]^{-1} \kappa\left(\lambda_{0}-z\right)^{-1} A P_{0}\right\} .
$$

Now, $A$ and $B$ are one-one on $\mathscr{R}\left(P_{0}\right)$ and $\mathscr{R}\left(\left[B P_{0}\right]^{*}\right)=\mathscr{R}\left(P_{0}\right)$ [2, p. 331]. We may therefore write (2.2) as a determinant on $\mathscr{R}\left(P_{0}\right)$, and then the factor $\left(\lambda_{0}-z\right)^{m}$ may be taken inside the $m \times m$ determinant to yield

$$
\begin{aligned}
& \Delta(z, \kappa) \\
& \quad=\operatorname{det}\left\{\left(\lambda_{0}-z\right) I_{m}+\kappa\left[B P_{0}\right]^{*} A P_{0}-\kappa^{2}\left[B P_{0}\right]^{*} Q_{c}^{+}(z) A P_{0}+O\left(\kappa^{3}\right)\right\}
\end{aligned}
$$

uniformly in $z$, where $I_{m}$ is the identity on $\mathscr{R}\left(P_{0}\right)$ and $\left[I+\kappa Q_{c}^{+}(z)\right]^{-1}$ has been expanded in a Neumann series.

The operator $V_{0}=\left[B P_{0}\right]^{*} A P_{0}$ maps $\mathscr{R}\left(P_{0}\right)$ into itself, and is essentially the compression of the perturbation $B^{*} A$ to $\mathscr{R}\left(P_{0}\right)$. Using (1.1), we find that for $x, y \in \mathscr{H}$

$$
\begin{aligned}
\left(V_{0} x, y\right) & =\left(\left[B P_{0}\right]^{*} A P_{0} x, y\right)=\left(A P_{0} x, B P_{0} y\right)=\left(B P_{0} x, A P_{0} y\right) \\
& =\left(\left[A P_{0}\right]^{*} B P_{0} x, y\right)=\left(V_{0}^{*} x, y\right)
\end{aligned}
$$

which means that $V_{0}$ is self-adjoint on $\mathscr{R}\left(P_{0}\right)$. Therefore, with respect to a suitable orthonormal basis $\phi_{1}, \cdots, \phi_{m}$ of $\mathscr{R}\left(P_{0}\right), V_{0}$ has a diagonal matrix 


$$
D=\left(\begin{array}{llll}
\lambda_{1} & & \\
& \lambda_{2} & & \\
& & \ddots & \\
& & & \lambda_{m}
\end{array}\right) .
$$

The perturbation $B^{*} A$ is said to remove the degeneracy at $\lambda_{0}$ iff the eigenvalues $\lambda_{1}, \cdots, \lambda_{m}$ to $V_{0}$ are all distinct. If $X(z)$ denotes the matrix with entries

$$
X_{i j}(z)=-\left(Q_{c}^{+}(z) A \phi_{i}, B \phi_{j}\right)
$$

then writing (2.3) with respect to the basis $\dot{\phi}_{1}, \cdots, \phi_{m}$ yields finally

$$
\Delta(z, \kappa)=\operatorname{det}\left\{\left(\lambda_{0}-z\right) I_{m}+\kappa D+\kappa^{2} X(z)+O\left(\kappa^{3}\right)\right\}
$$

uniformly in $z$ on a neighborhood of $\lambda_{0}$.

Theorem 2.1. If $B^{*} A$ removes the degeneracy at $\lambda_{0}$, then $z_{j}(\kappa)$ is analytic $(j=1, \cdots, m)$ and

$$
z_{j}(\kappa)=\lambda_{0}+\kappa \lambda_{j}+\kappa^{2} X_{j j}\left(\lambda_{0}\right)+O\left(\kappa^{3}\right) \text {. }
$$

Taking the imaginary part of (2.5) for real $\kappa$, we obtain formally

$$
\begin{aligned}
\Gamma_{j}(\kappa) & =-\operatorname{Im} z_{j}(\kappa)=-\kappa^{2} \operatorname{Im}\left(Q_{c}^{+}\left(\lambda_{0}\right) A \phi_{j}, B \dot{\phi}_{j}\right)+O\left(\kappa^{3}\right) \\
& =-\kappa^{2} \operatorname{Im}\left(R_{c}\left(\lambda_{0}+i 0\right) V \dot{\phi}_{j}, V \phi_{j}\right)+O\left(\kappa^{3}\right) \\
& =(2 i)^{-1} \kappa^{2}\left(\left[R_{c}\left(\lambda_{0}-i 0\right)-R_{c}\left(\lambda_{0}+i 0\right)\right] V \dot{\phi}_{j}, V \dot{\phi}_{j}\right)+O\left(\kappa^{3}\right)
\end{aligned}
$$

and hence finally

$$
\Gamma_{j}(\kappa)=\pi \kappa^{2}\left(\delta_{c}\left(T-\lambda_{0}\right) V \phi_{j}, V \dot{\phi}_{j}\right)+O\left(\kappa^{3}\right)
$$

where $V=B^{*} A=A^{*} B, R_{c}(z)=R(z)-\left(\lambda_{0}-z\right)^{-1} P_{0}$, and

$$
\delta_{c}(T-\lambda)=(2 \pi i)^{-1}\left[R_{c}(\lambda-i O)-R_{c}(\lambda+i O)\right] .
$$

Formula (2.6) is Fermi's Golden Rule.

Proof of Theorem 2.1. We already know that $z_{j}(\kappa)=\lambda_{0}+O(\kappa)$, and hence $X\left(z_{j}(\kappa)\right)=X\left(\lambda_{0}\right)+O(\kappa)$. If we define

$$
\zeta_{j}(\kappa)=\kappa^{-1}\left(z_{j}(\kappa)-\lambda_{0}\right) .
$$

Then the equation for $\zeta_{j}(\kappa)$ is, by $(2.4)$,

$$
\operatorname{det}\left\{-\kappa \zeta_{j}(\kappa) I_{m}+\kappa D+\kappa^{2} X\left(\lambda_{0}\right)+O\left(\kappa^{3}\right)\right\}=0 .
$$

Expanding and dividing by $\kappa^{m}$ gives

$$
\left(\lambda_{1}-\zeta_{j}(\kappa)\right) \cdots\left(\lambda_{m}-\zeta_{j}(\kappa)\right)+O(\kappa)=0 .
$$


Since the polynomial $\left(\lambda_{1}-\zeta\right) \cdots\left(\lambda_{m}-\zeta\right)$ obtained for $\kappa=0$ has distinct simple zeros, equation (2.8) has $m$ analytic solutions, one asymptotic to each root as $\kappa \rightarrow 0$. Thus we may take

$$
\zeta_{j}(\kappa)=\lambda_{j}+\beta_{j} \kappa+O\left(\kappa^{2}\right) \quad(j=1, \cdots, m) .
$$

Setting $j=1$ and substituting into (2.7), we find that

$$
\operatorname{det}\left\{\kappa J+\kappa^{2} X\left(\lambda_{0}\right)+O\left(\kappa^{3}\right)\right\}=0
$$

where

$$
J=\left(\begin{array}{lll}
-\kappa \beta_{1} & & \\
& \left(\lambda_{2}-\lambda_{1}\right)-\kappa \beta_{1} & \\
& \ddots & \\
& & \left(\lambda_{m}-\lambda_{1}\right)-\kappa \beta_{1}
\end{array}\right) .
$$

Expanding (2.7) gives

$$
\kappa^{m+1}\left(\lambda_{2}-\lambda_{1}\right) \cdots\left(\lambda_{m}-\lambda_{1}\right)\left(X_{11}\left(\lambda_{0}\right)-\beta_{1}\right)+O\left(\kappa^{m+2}\right)=0
$$

so that, in fact,

$$
\beta_{1}=X_{11}\left(\lambda_{0}\right)
$$

3. Spectral concentration. The following theorem extends the main result of [3] to embedded eigenvalues.

THEOREM 3.1. Assume that there exists a subspace $\mathscr{D}$ of $\mathscr{D}(A) \cap$ $\mathscr{D}(B)$ such that $B \mathscr{D} \subset \mathscr{D}\left(A^{*}\right), A \mathscr{D} \subset \mathscr{D}\left(B^{*}\right)$, and which is dense in $\mathscr{D}(A)$ and $\mathscr{D}(B)$ in the respective graph norms. For $j=1, \cdots, m$ and $\kappa$ real, choose $\delta_{j}(\kappa)$ such that $\delta_{j}(\kappa)=o(1)$ and $\operatorname{Im} z_{j}(\kappa)=o\left(\delta_{j}(\kappa)\right)$ as $\kappa \rightarrow 0$. Let

$$
S(\kappa)=\bigcup_{j=1}^{m}\left\{t: \operatorname{Re} z_{j}(\kappa)-\delta_{j}(\kappa)<t<\operatorname{Re} z_{j}(\kappa)+\delta_{j}(\kappa)\right\} .
$$

If $H(\kappa)=\int \lambda d E_{\kappa}(\lambda)$, then

$$
P_{0}=s t-\lim _{\kappa \rightarrow 0} \int_{S(\kappa)} d E_{\kappa}(\lambda)
$$

As shown in the appendix, the additional hypothesis insures that, for real $\kappa$, the poles of $Q_{1}^{+}(z, \kappa)$ are the complex conjugates of those of $Q_{1}^{-}(z, \kappa)$. Thus we did not need to take into account the poles of $Q_{1}^{-}(z, \kappa)$ when defining $S(\kappa)$, as was done for the corresponding set $J_{n}$ in [3, Theorem 2.1]. In order that $\mathscr{D}$ exists, it is sufficient that either $A$ or $B$ be bounded, or that $A$ and $B$ be commuting self-adjoint operators. 
Theorem 3.1 has a proof very similar to that of [3, Theorem 2.1], but cannot be deduced directly from that result because the operator $Q_{1}^{+}(z, \kappa)$, which corresponds to $Q_{1}^{+}(z, n)$ of [3], tends to zero as $\kappa \rightarrow 0$, and cannot, therefore, satisfy Hypothesis III (b) of [3]. To avoid repeating the lengthy arguments of [3], we shall simply carry the argument along to a point at which the arguments become essentially identical. A considerable study of [3] is therefore necessary to understanding the remainder of this section.

In order to surmount the difficulties posed by nonsimple poles, or poles close together, we shall show that for real $\kappa$, the resonances $z_{1}(\kappa), \cdots, z_{m}(\kappa)$ may be grouped into what we shall call clusters in such a way that, as $\kappa \rightarrow 0$, the resonances of a single cluster act together as a single, simple pole of $Q_{1}^{+}(z, \kappa)$, at least insofar as their asymptotic effect on the spectral measure of $H(\kappa)$ is concerned.

The result of our considerations is a rather detailed description of the singular part of $Q_{1}^{+}(z, \kappa)$.

In the first two lemmas, $\kappa$ may be complex.

Lemma 3.2. Let $z_{j}(\kappa)(j=1, \cdots, N)$ be the distinct poles of $Q_{1}^{+}(z, \kappa)$. Then $Q_{1}^{+}(z, \kappa)$ has the partial fraction expansion

$$
Q_{1}^{+}(z, \kappa)=\sum_{j=1}^{N} \frac{B_{1}^{(j)}(\kappa)}{\left(z-z_{j}(\kappa)\right)}+\cdots+\frac{B_{m_{j}}^{(j)}(\kappa)}{\left(z-z_{j}(\kappa)\right)^{m_{j}}}+L(z, \kappa),
$$

where $L(z, \kappa)$ is analytic in $z$ and $\kappa$. If $z_{j}(\kappa)$ has a Puiseux series expansion in powers of $\kappa^{1 / p}$, then $B_{k}^{(j)}(\kappa)\left(k=1, \cdots, m_{j}\right)$ also has an expansion in powers of $\kappa^{1 / p}$, and has at most an algebraic pole at $\kappa=0$.

The proof is a simple adaptation of the argument on pp. 69-70 of [6]. Certain additional facts obtained there do not hold here, since $Q_{1}^{+}(z, \kappa)$ is not a resolvent. Analyticity of $L(z, \kappa)$ is proved in the proof of the next lemma.

It follows immediately that for small $\kappa \neq 0, B_{\kappa}^{(j)}(\kappa)$ either vanishes identically or is never zero. Hence, for small $\kappa \neq 0$, the order $m_{j}$ of the $j$ th pole $z_{j}(\kappa)$ of $Q_{1}^{+}(z, \kappa)$ is independent of $\kappa$.

If the terms of the singular part of $Q_{1}^{+}(z, \kappa)$ in (3.1) are combined, we obtain

$$
Q_{1}^{+}(z, \kappa)=\frac{P(z, \kappa)}{\Delta(z, \kappa)}+L(z, \kappa)
$$

where $P(z, \kappa)$ is a polynomial in $z$ with coefficients having at most an algebraic singularity at $\kappa=0$, and $\Delta(z, \kappa)$ is the analytic function of $z$ and $\kappa$ defined in $\S 1$. 
LEMma 3.3. (a) As $\kappa \rightarrow 0, Q_{1}^{+}(z, \kappa) \rightarrow Q^{+}(z)$ uniformly on $0<$ $\varepsilon \leqq\left|z-\lambda_{0}\right| \leqq \delta_{2}$ for every $\varepsilon>0$.

(b) $P(z, \kappa), \Delta(z, \kappa)$, and $L(z, \kappa)$ are all analytic in $z$ and $\kappa$. Moreover,

$$
\lim _{\kappa \rightarrow 0} P(z, \kappa)=\left(z-\lambda_{0}\right)^{N-1} A P_{0}\left[B P_{0}\right]^{*}
$$

Proof. From (1.3) and (2.1) one obtains

$$
I-\kappa Q_{1}^{+}(z, \kappa)=\left[I+\kappa\left(\lambda_{0}-z\right)^{-1} \Gamma(z, \kappa) A P_{0}\left[B P_{0}\right]^{*}\right]^{-1} \Gamma(z, \kappa)
$$

where

$$
\Gamma(z, \kappa)=\left[I+\kappa Q_{c}^{+}(z)\right]^{-1}
$$

is analytic in $z$ and $\kappa$, for $\kappa$ and $z-\lambda_{0}$ small. Expanding the right side, canceling $I$ on both sides and dividing by $\kappa$ yields the result. Analyticity of $L(z, \kappa)$ and the coefficients of $P(z, \kappa)$, as well as (3.2) follow from the formulas between equations (2.7) and (2.8) of [3], where the discrete parameter $n$ must be replaced by $\kappa$.

Assume now that $\kappa$ is real, and write

$$
z_{j}(\kappa)=\lambda_{j}(\kappa)-i \Gamma_{j}(\kappa) \quad(j=1, \cdots, N)
$$

where $\lambda_{j}(\kappa)$ is real and $\Gamma_{j}(\kappa) \geqq 0$. We shall now describe the grouping of the $z_{j}(\kappa)$ 's into clusters. To begin with, we specify that if $\Gamma_{j}(\kappa) \equiv 0$, then $z_{j}(\kappa)$ is to form a cluster by itself. Otherwise, $\Gamma_{j}(\kappa)>$ 0 for small $\kappa \neq 0$, and we shall assume now for convenience that

$$
\Gamma_{j}(\kappa)>0 \quad(j=1, \cdots, N) .
$$

Then $\Gamma_{j}(\kappa)$ has a Puiseux series, so that

$$
\Gamma_{j}(\kappa)=a_{j} \kappa^{p(j)}+\cdots
$$

where $a_{j}>0$ and $p(j)$ is an integer $(j=1, \cdots, m$ ). (If $\kappa$ is complex in (3.4), $\Gamma_{j}(\kappa)$ is defined, but no longer the imaginary part of $-z_{j}(\kappa)$.) For $\kappa \neq 0$, choose $\delta_{j}(\kappa)>0$ such that

$$
\delta_{j}(\kappa)=o\left(\kappa^{p(j)-1}\right) \quad(j=1, \cdots, m)
$$

while

$$
\kappa^{p(j)}=o\left(\delta_{j}(\kappa)\right)
$$$$
(j=1, \cdots, m)
$$

as $\kappa \rightarrow 0$, and consider the intervals

$$
J_{j}(\kappa)=\left(\lambda_{j}(\kappa)-\delta_{j}(\kappa), \lambda_{j}(\kappa)+\delta_{j}(\kappa)\right) .
$$

If $\kappa$ is small, the number of component intervals of 


$$
J_{1}(\kappa) \cup \cdots \cup J_{m}(\kappa)
$$

is independent of $\kappa$, and each component is the union of the intervals $J_{j}(\kappa)$ corresponding to a certain set of resonances. For the distance between $\lambda_{j}(\kappa)$ and $\lambda_{k}(\kappa)$ is of the order of some integral power of $\kappa$, and is therefore either much greater or much less than the length of $J_{j}(\kappa)$. These sets are the clusters; they are independent of $\kappa$. We shall denote the components of (3.5) by

$$
\left(c_{j}(\kappa)-\rho_{j}(\kappa), c_{j}(\kappa)+\rho_{j}(\kappa)\right) \quad(j=1, \cdots, N)
$$

where $N$ is the number of clusters. We shall refer to $c_{j}(\kappa)$ and $\rho_{j}(\kappa)$ as the center and radius of the $j$ th cluster.

It is easily seen that if $\left\{z_{1}(\kappa), \cdots, z_{p_{1}}(\kappa)\right\}$ is the first cluster, then

$$
\lambda_{j}(\kappa)-c_{1}(\kappa)=o\left(\rho_{1}(\kappa)\right)
$$$$
\left(j=1, \cdots, p_{1}\right) \text {. }
$$

For if $\lambda_{j}(\kappa)$ and $\lambda_{k}(\kappa)$ belong to the first cluster, the distance between them is much less than either $\delta_{j}(\kappa)$ or $\delta_{k}(\kappa)$, neither of which can exceed $\rho_{1}(\kappa)$. Similarly

$$
\rho_{i}(\kappa)=o\left(\left|c_{1}(\kappa)-c_{2}(\kappa)\right|\right)
$$

because $c_{1}(\kappa)-c_{2}(\kappa)$, being determined by the $\lambda_{j}(\kappa)$ 's, is of integral power order, while $\rho_{j}(\kappa)$, being determined by the $\delta_{j}(\kappa)$ 's is not.

Similar statements hold for other clusters. The interpretation of (3.6) is that the resonances of a cluster are asymptotically very close to the center of the corresponding interval $\left(c_{n}-\rho_{n}, c_{n}+\rho_{n}\right)$, while (3.7) says that distinct components of (3.5) are asymptotically very small compared to their distance apart.

LeMma 3.4. For $\operatorname{Im} z>0$, and $\left|z-\lambda_{0}\right| \leqq \delta_{2}$

$$
\|P(z, \kappa)\| \leqq C|\Delta(z, \kappa)|(\operatorname{Im} z)^{-1}
$$

where $C$ is independent of $\kappa$.

Proof. For each $\kappa$, the coefficients of $P(z, \kappa)$ are of finite rank, since they are residues of functions with singular parts of finite rank, and are also analytic in $\kappa$. The lemma therefore follows by a proof similar to that of equation (2.8) of [3].

The procedures of [3] could now be applied to yield an asymptotic expansion for the singular part $P(z, \kappa) / \Delta(z, \kappa)$ of $Q_{1}^{+}(z, \kappa)$. However, we shall be content to remark that for any sequence $\kappa_{n} \rightarrow 0$, the quantities $P\left(z, \kappa_{n}\right), \Delta\left(z, \kappa_{n}\right)$, etc. have precisely the properties of $P_{n}(z)$, $\Delta_{n}(z)$ etc. which are used in the proof of [3, Theorem 2.1] from equation (2.10) of [3] onward. The remainder of the proof of Theorem 3.1 follows [3] with essentially no change. 
4. Examples. We shall now consider some simple examples which illustrate certain phenomena.

EXAMPLE 1. We shall first give an example in which a nonanalytic Puiseux series occurs. Let $\mathscr{H}=L_{2}(-\infty,+\infty) \oplus \mathscr{C}^{2}$, and let $e_{1}, e_{2}$ be the usual orthonormal basis of $\mathscr{C}^{2}$. Define

$$
H_{0}\left(\begin{array}{c}
u(t) \\
\xi
\end{array}\right)=\left(\begin{array}{ll}
t & 0 \\
0 & c
\end{array}\right)\left(\begin{array}{c}
u(t) \\
\xi
\end{array}\right)=\left(\begin{array}{c}
t u(t) \\
c \xi
\end{array}\right)
$$

where $u \in L_{2}(-\infty,+\infty), \xi \in \mathbb{C}^{2}$ and $c$ is a fixed real number. $H_{0}=T$ has absolutely continuous spectrum of simple multiplicity, except for an embedded eigenvalue $c$ of multiplicity $m=2$. Let $f_{1}(t), f_{2}(t)$ be an orthonormal pair of functions in $L_{2}(-\infty,+\infty)$, and define an operator $Y$ from $\ell^{2}$ into $L_{2}(-\infty,+\infty)$ by

$$
Y\left(\xi_{1} e_{1}+\xi_{2} e_{2}\right)=\xi_{1} f_{1}(t)+\xi_{2} f_{2}(t) .
$$

The operator $Y^{*}$ from $L_{2}(-\infty,+\infty)$ back into $\mathbb{C}^{2}$ is then

$$
Y^{*} u=\left(\int u(t) \bar{f}_{1}(t) d t\right) e_{1}+\left(\int u(t) \bar{f}_{2}(t) d t\right) e_{2} .
$$

We shall consider the perturbed operator

$$
H(\kappa)=H_{0}+\kappa V
$$

where

$$
V=\left(\begin{array}{cc}
0 & Y \\
Y^{*} & \lambda_{1} I
\end{array}\right)
$$

and $\lambda_{1}>0$. The perturbation $V$ is self-adjoint of rank 4 , and its range has the orthonormal basis $\left\{f_{1}, f_{2}, e_{1}, e_{2}\right\}$. If we choose the factorization

$$
V=V P=P V
$$

where $P$ is the orthogonal projection onto the range of $V$, then the matrix of

$$
Q(z)=V\left(H_{0}-z\right)^{-1} P
$$

with respect to the orthonormal basis $f_{1}, f_{2}, e_{1}, e_{2}$ of the range of $V$ is

$$
\left(\begin{array}{cc}
0 & (c-z)^{-1} I_{2} \\
F(z) & (c-z)^{-1} \lambda_{1} I_{2}
\end{array}\right)
$$

where 


$$
F(z)=\int(t-z)^{-1}\left(\begin{array}{cc}
\left|f_{1}(t)\right|^{2} & \bar{f}_{1}(t) f_{2}(t) \\
f_{1}(t) \bar{f}_{2}(t) & \left|f_{2}(t)\right|^{2}
\end{array}\right) d t
$$

and $I_{2}$ is the $2 \times 2$ identity matrix.

If we now assume that $F(z)$ has a meromorphic continuation from the upper half-plane across the axis in a neighborhood of $c$, then the equation

$$
(c-z)^{2} \operatorname{det}(I+\kappa Q(z))=0
$$

for the resonances reduces to

$$
\kappa^{4} D(z)-\kappa^{2} T(z)\left(c+\kappa \lambda_{1}-z\right)+\left(c+\kappa \lambda_{1}-z\right)^{2}=0
$$

where $T(z)$ and $D(z)$ are the trace and determinant of $F(z)$. Solving for $\left(c+\kappa \lambda_{1}-z\right)^{-1}$ by the quadratic formula yields

$$
z=c+\lambda_{1} \kappa+\kappa^{2} g(z)
$$

where

$$
g(z)=-\frac{1}{2}\left(T(z) \pm \sqrt{T^{2}(z)-4 D(z)}\right) .
$$

For simplicity, let us now take $c=0$. Then, if the function

$$
H(z)=T^{2}(z)-4 D(z)
$$

has a simple zero at $z=0$, the function $g(z)$ has a Puiseux series expansion

$$
g(z)=a_{0}+a_{1} z^{1 / 2}+a_{2} z+\cdots
$$

where $a_{1} \neq 0$. It then follows easily from

$$
z=\lambda_{1} \kappa+\kappa^{2}\left(a_{0}+a_{1} z^{1 / 2}+a_{2} z+\cdots\right)
$$

that

$$
z=\lambda_{1} \kappa+a_{0} \kappa^{2}+a_{1} \lambda_{1}^{1 / 2} \kappa^{5 / 2}+O\left(\kappa^{3}\right)
$$

which means that $z(\kappa)$ has a nonanalytic Puiseux series in $\kappa$. We shall therefore have obtained the desired example, if we can find $f_{1}(t)$ and $f_{2}(t)$ such that $H(z)$ has a simple zero at $z=0$.

To this end, let

$$
f_{1}(t)=\left(\frac{2}{\pi}\right)^{1 / 2} \frac{1}{t^{2}+1}
$$

and

$$
\begin{aligned}
f_{2}(t) & =(2-2 \varepsilon)^{-1 / 2} \operatorname{sgn} t & & 0<\varepsilon<|t|<1 \\
& =0 & & \text { otherwise . }
\end{aligned}
$$


Then $f_{1}$ and $f_{2}$ are an orthonormal pair, and since they are real,

$$
F_{12}(z)=F_{21}(z) \text {. }
$$

The values of $F_{11}(0)$ and $F_{11}^{\prime}(0)$ may be computed from

$$
F_{11}(z)=-(z+2 i)(z+i)^{-2} \quad \operatorname{Im} z>0
$$

while due to the fact that $f_{2}(t)$ vanishes near the origin, the integrals for $F_{12}(0)$ and $F_{22}(0)$, as well as those obtained for $F_{12}^{\prime}(0)$ and $F_{22}^{\prime}(0)$ by differentiation under the integral sign are absolutely convergent. In fact, one has

$$
F_{22}(0)=(2-2 \varepsilon)^{-1} \int_{\varepsilon<|t|<1} \frac{d t}{t}=0
$$

and

$$
F_{22}^{\prime}(0)=(2-2 \varepsilon)^{-1} \int_{\varepsilon<|t|<1} \frac{d t}{t^{2}}=\varepsilon^{-1}
$$

Similarly,

$$
F_{12}(0)=2(\pi-\pi \varepsilon)^{-1 / 2} \int_{\varepsilon}^{1} \frac{1}{t^{2}+1} \cdot \frac{d t}{t}
$$

and

$$
F_{12}^{\prime}(0)=0
$$

Hence, one computes that

$$
\begin{aligned}
H(0) & =\left(F_{11}(0)-F_{22}(0)\right)^{2}+4 F_{12}^{2}(0) \\
& =-4+16(\pi-\pi \varepsilon)^{-1}\left\{\int_{\varepsilon}^{1} \frac{1}{t^{2}+1} \frac{d t}{t}\right\}^{2}
\end{aligned}
$$

and

$$
\begin{aligned}
H^{\prime}(0) & =2\left(F_{11}(0)-F_{22}(0)\right)\left(F_{11}^{\prime}(0)-F_{22}^{\prime}(0)\right)+8 F_{12}(0) F_{12}^{\prime}(0) \\
& =-4 i\left(3+\varepsilon^{-1}\right) \neq 0 .
\end{aligned}
$$

It therefore remains to choose $\varepsilon$ such that $H(0)=0$; that is, such that

$$
\left(\frac{\pi}{4}\right)^{1 / 2}=(1-\varepsilon)^{-1 / 2} \int_{\varepsilon}^{1} \frac{1}{t^{2}+1} \frac{d t}{t} \equiv \Phi(\varepsilon) .
$$

But since $\Phi(\varepsilon)$ is decreasing on $0<\varepsilon<1, \Phi(0+)=+\infty$, and $\Phi(1-)=$ 0 , there is a unique $\varepsilon$ in the interval $0<\varepsilon<1$ satisfying this equation.

Finally, note that the Puiseux series appears here as a degenerate case, since in the usual case when $H(z)$ does not vanish at the origin, $g(z)$ and hence $z(\kappa)$, have two distinct analytic branches. 
EXAMPLE 2. An example will now be given of an eigenvalue of multiplicity one embedded at an end point of the continuous spectrum, and perturbed by an operator of rank two, which gives rise to a resonance or an eigenvalue which cannot be represented as a Puiseux series. The endpoint appears as a branch point of $Q^{+}(z)$. Branch points of continued quantities occur in Simon's articles [14, 15] as "thresholds" for certain processes (that is, the minimum energies at which the processes can occur). His theory excludes eigenvalues embedded at thresholds-with good reason, as this example shows. Most of the thresholds in $[14,15]$ are embedded in a continuous spectrum, rather than at an end point. An example of this along the present lines would be easily constructed. The example is similar to Example 8.3 of [5, p. 581]. The operator $H_{0}=T$ on $L_{2}(0, \infty) \oplus$ $C$ defined by

$$
H_{0}[u(t), \xi]=[t u(t), 0]
$$

has absolutely continuous spectrum $[0, \infty)$ and an eigenvalue at $\lambda_{0}=0$ with eigenvector

$$
\phi_{0}=[0,1] .
$$

Let $H(\kappa)=H_{0}+\kappa V$ where

$$
V[u(t), \xi]=\left[\xi f(t),(u, f)+\lambda_{1} \xi\right] .
$$

We assume that $\lambda_{1}>0$ and

$$
\int_{0}^{\infty}|f(t)|^{2} d t=1
$$

The perturbation $V$ has rank 2, so the resonances are to be sought as poles of an analytic continuation of the inverse of the matrix $W(z, \kappa)$ of the restriction of $I+\kappa V\left(H_{0}-z\right)^{-1}$ to the range $\mathscr{R}(V)$ of $V$. Computing $W(z, \kappa)$ with respect to the orthonormal basis $\phi_{0}, f$ of $\mathscr{R}(V)$, one obtains [5; eq. (8.9), p. 581]

$$
W(z, \kappa)=\left(\begin{array}{cc}
1 & -\kappa z^{-1} \\
\kappa F(z) & 1-\kappa \lambda_{1} z^{-1}
\end{array}\right)
$$

where

$$
F(z)=\int_{0}^{\infty}|f(t)|^{2}(t-z)^{-1} d t
$$

If we assume that $F(z)$ has a continuation $F_{+}(z)$ from the upper halfplane across the positive real axis, then the resonances satisfy the equation

$$
z=\kappa \lambda_{1}-\kappa^{2} F_{+}(z) .
$$


(See [5, p. 581], the third equation from the bottom of the page-in which there is an error of sign.)

Now choose

$$
|f(t)|^{2}=\frac{2}{\pi} \frac{1}{1+t^{2}}
$$

so that

$$
F(z)=\frac{2 i-(2 / \pi) \log z-z}{1+z^{2}}
$$

where $0<\arg z<2 \pi$. The solution of (4.1) then has the asymptotic expansion

$$
z(\kappa)=\kappa \lambda_{1}+(2 / \pi) \kappa^{2} \log \left(\kappa \lambda_{1}\right)-2 i \kappa^{2}+O\left(\kappa^{3}\right)
$$

which is not of Puiseux type. For $\kappa<0, z(\kappa)$ lies in the region $0<$ $\arg z<2 \pi$, and is therefore a negative eigenvalue $\lambda(\kappa)$ of $H(\kappa)$, with the expansion

$$
\lambda(\kappa)=\kappa \lambda_{1}+(2 / \pi) \kappa^{2} \log \left(-\kappa \lambda_{1}\right)+O\left(\kappa^{3}\right) \kappa<0 .
$$

For $\kappa>0$, the continuation $F_{+}(z)$ of $F(z)$ leads to the solution $z_{+}(\kappa)$ with $\arg z_{+}(\kappa) \cong 0$, while if $F_{+}(z)$ is replaced in (4.1) by the continuation $F_{-}(z)$ of $F(z)$ from the lower half-plane, one obtains the solution $z_{-}(\kappa)$ with $\arg z_{-}(\kappa) \cong 2 \pi$. These numbers are complex conjugates. If $\kappa$ is complex, the first situation essentially prevails, in the sense that the non-self-adjoint operator $H(\kappa)$ has an eigenvalue at $z(\kappa)$ for all sufficiently small $\kappa$ in any given sector $|\arg \kappa-\pi| \leqq$ $\pi-\delta, \delta>0$.

If instead of (4.2), one chooses

$$
|f(t)|^{2}=\frac{2}{\pi} \cos (\pi \alpha / 2) \frac{t^{\alpha}}{1+t^{2}}
$$

where $-1<\alpha<1$, then one obtains, for $\alpha \neq 0$,

$$
F(z)=\frac{\cot (\pi \alpha / 2)-\csc (\pi \alpha / 2) z^{\alpha} e^{-i \pi \alpha}-z}{1+z^{2}}
$$

where $0<\arg z<2 \pi$. The solution of (4.2) then has the expansion

$$
z(\kappa)=\kappa \lambda_{1}-\kappa^{2} \cot (\pi \alpha / 2)+\kappa^{2+\alpha} e^{-i \pi \alpha} \lambda_{1}^{\alpha} \csc (\pi \alpha / 2)+O\left(\kappa^{3}\right) .
$$

This has the same general behavior: for $\kappa>0$, there is an eigenvalue $\lambda(\kappa)$ with expansion

$$
\lambda(\kappa)=\kappa \lambda_{1}-\kappa^{2} \cot (\pi \alpha / 2)+(-\kappa)^{2+\alpha} \lambda_{1}^{\alpha} \csc (\pi \alpha / 2)+O\left(\kappa^{3}\right)
$$

while for $\kappa>0$, there is a resonance. A notable feature, however, 
is that one may obtain a Puiseux series by taking, for example, $\alpha=$ $\pm 1 / 2$, in which case $W(z, \kappa)$ has only an algebraic singularity at $z=$ 0 . In fact there are only two sheets, and it is interesting to note that for $\kappa<0$, these is a pole on the second sheet directly below the eigenvalue $\lambda(\kappa)$.

Let us see what becomes of Fermi's Golden Rule in this case. One has

$$
\left\langle\delta_{c}\left(H_{0}-\lambda\right) \bar{V} \phi_{0}, \bar{V} \dot{\phi}_{0}\right\rangle=|f(\lambda)|^{2} .
$$

(See [5, eq. (8.7)]. Note that, in the notation of [5], the $V_{1}$ term contributes nothing.) Hence, Fermi's Rule gives

$$
\Gamma(\kappa) \cong \pi \kappa^{2}\left|f\left(\lambda_{0}\right)\right|^{2}
$$

Applied to the case $\lambda_{0}=0$ with $f(t)$ given by (4.4), this gives the following results: (a) for $\alpha=0$

$$
\Gamma(\kappa) \cong 2 \kappa^{2}
$$

which agrees with (4.3); (b) for $\alpha>0$

$$
\Gamma(\kappa) \cong 0
$$

which agrees with (4.5), to order $\kappa^{2}$, but is not informative; (c) for $\alpha<0, \Gamma(\kappa)$ is infinite, which is not surprising because according to (4.5), $\Gamma(\kappa)$ is not $O\left(\kappa^{2}\right)$. The Gold from which the Rule is made is apparently mixed with Brass.

If, however, $\lambda_{0}$ is replaced in the Rule by $\lambda_{0}+\kappa \lambda_{1}$, the resulting formula

$$
\Gamma(\kappa) \cong \pi \kappa^{2}\left\langle\delta_{c}\left(H_{0}-\lambda_{0}-\kappa \lambda_{1}\right) V \phi_{0}, V \phi_{0}\right\rangle
$$

is an unalloyed success; for one then obtains

$$
\Gamma(\kappa) \cong \pi \kappa^{2}\left|f\left(\kappa \lambda_{1}\right)\right|^{2} \cong 2 \lambda_{1}^{\alpha} \kappa^{2+\alpha} \cos (\pi \alpha / 2)
$$

which agrees with (4.5).

APPENDIX. Let $T$ be self-adjoint and suppose that for some pair of vectors $f, g$ the function

$$
r(z)=\left((T-z)^{-1} f, g\right)
$$

has meromorphic continuations $r_{ \pm}(z)$ across some interval of the real axis. That the poles of $r_{-}(z)$ need not be the complex conjugates of the poles of $r_{+}(z)$ may be seen by taking $T u(t)=t u(t)$ on $L_{2}(-\infty,+\infty)$ and choosing $f(t)=(t+i)^{-1}$ and $g(t)=(t-i)^{-1}$. Then $r_{+}(z)$ has a pole at $z=-i$, while $r_{-}(z)$ vanishes identically. 
Similarly, the poles of $Q_{1}^{+}(z)$ and $Q_{1}^{-}(z)$ are not always conjugate. For $A=(\cdot, f) f$ and $B=(\cdot, g) g$ are bounded and self-adjoint, and $A B=B A=0$ because $f$ and $g$ are orthogonal. Hence, $H=T+$ $B^{*} A=T$, and

$$
Q_{1}(z)=Q(z)=(G(z) f, g)(\cdot, g) g=r(z)(\cdot, f) g
$$

so that $Q_{1}^{+}(z)$ has a pole at $z=-i$ while $Q_{1}^{-}(z)$ vanishes identically.

We shall give sufficient conditions that $Q_{1}^{+}(z)$ and $Q_{1}^{-}(z)$ have conjugate poles. Let $T, A$, and $B$ satisfy the hypotheses of $\S 1$, and assume that $Q_{1}^{ \pm}(z)$ defined by

$$
I-Q_{1}^{ \pm}(z)=\left[I+Q^{ \pm}(z)\right]^{-1}
$$

is meromorphic, and has finite rank principal parts at all its poles. This is true, for example, if $\kappa$ is small in $\S 1$, or if $Q^{ \pm}(z)$ is compact. Formula (1.2) (with $\kappa=1$ ) then defines the resolvent $R(z)$ of an extension $H$ of $T+B^{*} A$, and $Q_{1}(z)$ is the extension of $A R(z) B^{*}$. (It is not clear whether or not $H$ is self-adjoint in this generality, but this is not at issue.) By taking adjoints, [7, eq. (2.2)] one also finds that $B G(z) A^{*}$ has the compact extension

$$
\widetilde{Q}(z)=[Q(\bar{z})]^{*}
$$

which has the continuations

$$
\widetilde{Q}^{ \pm}(z)=\left[Q^{ \pm}(z)\right]^{*}
$$

defined on $\Omega$. Similarly, $B R(z) A^{*}$ leads to $\widetilde{Q}_{1}(z)$ and $\widetilde{Q}_{1}^{ \pm}(z)$.

THeOREM. In addition to the hypotheses above, suppose that there exists a subspace $\mathscr{D}$ of $\mathscr{D}(A) \cap \mathscr{D}(B)$ such that $B \mathscr{D} \subset \mathscr{D}\left(A^{*}\right)$, $A \mathscr{D} \subset \mathscr{D}\left(B^{*}\right)$, and $\mathscr{D}$ is dense in $\mathscr{D}(A)$ and $\mathscr{D}(B)$ respectively, in the graph norms. If $Q^{+}(z)$ is analytic at $z_{0}$, then $Q_{1}^{+}(z)$ is analytic at $z_{0}$ iff $\widetilde{Q}_{1}^{+}(z)$ is analytic at $z_{0}$.

Proof. Let $P_{A}$ and $P_{B}$ be the orthogonal projections onto the closures of the ranges of $A$ and $B$. Then $I-P_{B}$ projects onto ker $B^{*}$, so that

$$
P_{A} Q(z)=Q(z) \text { and } Q(z)\left[I-P_{B}\right]=0
$$

for $\operatorname{Im} z>0$, and hence by continuation

$$
P_{A} Q^{+}(z)=Q^{+}(z)
$$

and

$$
Q^{+}(z) P_{B}=Q^{+}(z)
$$


Observe next that by (1.1),

$$
B^{*} A x=A^{*} B x \quad x \in \mathscr{D} .
$$

Hence, for $x, g \in \mathscr{D}$, and $\operatorname{Im} z>0$, one has

$$
\begin{aligned}
& \left(\widetilde{Q}_{1}(z) B x, A y\right)=\left(B R(z) A^{*} B x, A y\right) \\
& \quad=\left(B R(z) B^{*} A x, A y\right)=\left(A R(z) B^{*} A x, B y\right) \\
& \quad=\left(Q_{1}(z) A x, B y\right)
\end{aligned}
$$

where (1.1) was used in the equality next to last. Using that $\mathscr{D}$ is dense in the graphs, and passing to a continuation shows that analyticity of $P_{A} \widetilde{Q}_{1}^{+}(z) P_{B}$ at $z_{0}$ is equivalent to analyticity of $P_{B} Q_{1}^{+}(z) P_{A}$ at $z_{0}$.

If we now assume that $Q^{+}(z)$ and $\widetilde{Q}_{1}^{+}(z)$ are analytic at $z_{0}$, then since (1), together with (2) and (3), implies that

$$
\begin{aligned}
Q_{1}^{+}(z) & =Q^{+}(z)-\left[Q^{+}(z)\right]^{2}+Q^{+}(z) Q_{1}^{+}(z) Q^{+}(z) \\
& =Q^{+}(z)-\left[Q^{+}(z)\right]^{2}+Q^{+}(z) P_{B} Q_{1}^{+}(z) P_{A} Q^{+}(z)
\end{aligned}
$$

it follows that $Q_{1}^{+}(z)$ is also analytic at $z_{0}$. The other implication is proved similarly.

It is evident from the proof that if the ranges of $A$ and $B$ are dense, the assumption that $Q^{+}(z)$ is analytic at $z_{0}$ may be dropped. However, the example above shows that it cannot be dropped in general.

COROLlaRY. If all poles of $Q^{+}(z)$ are real, then the nonreal poles of $Q_{1}^{+}(z)$ and $Q_{1}^{-}(z)$ are complex conjugates.

This follows from (1).

Proposition. Either of the following conditions suffices for the existence of $\mathscr{D}$.

(a) Either $A$ or $B$ is bounded.

(b) $A$ and $B$ are commuting self-adjoint operators.

Proof. If $A$ is bounded, it follows from (1.1) that $A \mathscr{D}(B) \subset$ $\mathscr{D}\left(B^{*}\right)$. Hence, one may take $\mathscr{D}=\mathscr{D}(B)$. Similarly if $B$ is bounded.

Sufficiency of (b) follows easily from [12, p. 358].

\section{REFERENCES}

1. S. Bochner and W. T. Martin, Several Complex Variables, Princeton University Press, 1948.

2. J. S. Howland, On the Weinstein-Aronszajn formula, Arch. Rat. Mech. Anal., 39 (1970), 323-339. 
3. J. S. Howland, Spectral concentration and virtual poles, II, Trans. Amer. Math. Soc., 162 (1971), 141-156.

4. , Perturbation of embedded eigenvalues, Bull. Amer. Math. Soc., 78 (1972), $280-283$.

5. — Embedded eigenvalues and virtual poles, Pacific J. Math., 29 (1969), $565-582$.

6. T. Kato, Perturbation Theory for Linear Operators, Berlin-Heidelberg-New York, Springer, 1966.

7. - Wave operators and similarity for some non-self-adjoint operators, Math. Ann., 162 (1966), 258-279.

8. T. Kato and S. T. Kuroda, The abstract theory of scattering, Rocky Mountain J. Math., 1 (1971), 127-171.

9. K. Knopp, Theory of Functions, Part II, New York, Dover, 1947.

10. R. Konno and S. T. Kuroda, On the finiteness of perturbed eigenvalues, J. Fac.

Sci. Univ. Tokyo Sect. I, 13 (1966), 55-63.

11. P. D. Lax and R. S. Phillips, Scattering Theory, Academic Press, New YorkLondon, 1967.

12. F. Riesz and B. Sz-Nagy, Functional Analysis, Ungar, New York, 1955.

13. N. A. Shenk and D. Thoe, Eigenfunction expansions and scattering theory for perturbations of -4 , Rocky Mountain J. Math., 1 (1971), 89-125.

14. B. Simon, Convergence of time dependent perturbation theory for autoionizing states of atoms, Phys. Letters A., 36 (1971), 23-24.

15. - Resonances in $N$-body quantum systems with dilatation analytic potentials and the foundations of time-dependent perturbation theory, Ann. Math., 97 (1973), 247-274.

Received March 23, 1973. Supported by DA-ARO-31-124-71-G182.

UNIVERSITY OF VIRGINIA 


\section{PACIFIC JOURNAL OF MATHEMATICS}

EDITORS

RICHARD ARENS (Managing Editor)

University of California

Los Angeles, Calıfornia 90024

R. A. Beaumont

University of Washington

Seattle, Washington 98105
J. DugundJI

Department of Mathematics

University of Southern California

Los Angeles, California 90007

D. Gilbarg and J. Milgram

Stanford University

Stanford, California 94305

\section{ASSOCIATE EDITORS}
E. F. BECKENBACH
B. H. NeumanN
F. WOLF
K. YOSHIDA

\section{SUPPORTING INSTITUTIONS}

UNIVERSITY OF BRITISH COLUMBIA

CALIFORNIA INSTITUTE OF TECHNOLOGY

UNIVERSITY OF CALIFORNIA

MONTANA STATE UNIVERSITY

UNIVERSITY OF NEVADA

NEW MEXICO STATE UNIVERSITY

OREGON STATE UNIVERSITY

UNIVERSITY OF OREGON

OSAKA UNIVERSITY
UNIVERSITY OF SOUTHERN CALIFORNIA

STANFORD UNIVERSITY

UNIVERSITY OF TOKYO

UNIVERSITY OF UTAH

WASHINGTON STATE UNIVERSITY

UNIVERSITY OF WASHINGTON

AMERICAN MATHEMATICAL SOCIETY NAVAL WEAPONS CENTER 


\section{Pacific Journal of Mathematics}

\section{Vol. 55, No. $1 \quad$ September, 1974}

Robert Lee Anderson, Continuous spectra of a singular symmetric

differential operator on a Hilbert space of vector-valued functions . . . $\quad 1$

Michael James Cambern, The isometries of $L^{p}(X, K) \ldots \ldots \ldots \ldots \ldots . . \ldots$

R. H. Cameron and David Arne Storvick, Two related integrals over spaces of continuous functions ................................

Gary Theodore Chartrand and Albert David Polimeni, Ramsey theory and

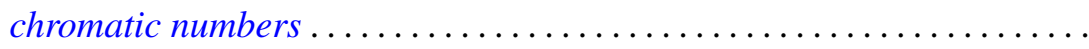

John Deryck De Pree and Harry Scott Klein, Characterization of collectively compact sets of linear operators ...................

John Deryck De Pree and Harry Scott Klein, Semi-groups and collectively compact sets of linear operators ....................... 55

George Epstein and Alfred Horn, Chain based lattices.............. 65

Paul Erdős and Ernst Gabor Straus, On the irrationality of certain series . . 85

Zdeněk Frolík, Measurable uniform spaces................... 93

Stephen Michael Gagola, Jr., Characters fully ramified over a normal

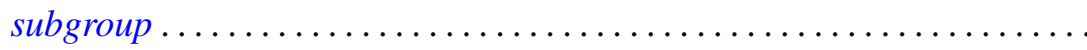

Frank Larkin Gilfeather, Operator valued roots of abelian analytic

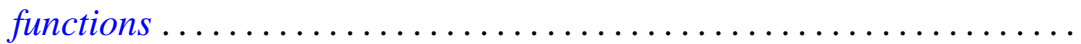

D. S. Goel, A. S. B. Holland, Cyril Nasim and B. N. Sahney, Best approximation by a saturation class of polynomial operators

James Secord Howland, Puiseux series for resonances at an embedded

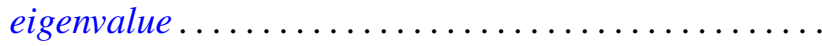

David Jacobson, Linear GCD equations .................

P. H. Karvellas, A note on compact semirings which are multiplicative

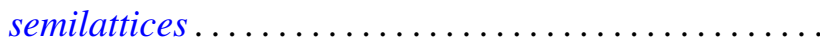

Allan Morton Krall, Stieltjes differential-boundary operators. II . .

D. G. Larman, On the inner aperture and intersections of convex sets

S. N. Mukhopadhyay, On the regularity of the $P^{n}$-integral and its application to summable trigonometric series ....... .

Dwight Webster Read, On $(J, M, m)$-extensions of Boolean algebras ....

David Francis Rearick, Multiplicativity-preserving arithmetic power series.

Indranand Sinha, Characteristic ideals in group algebras

Charles Thomas Tucker, II, Homomorphisms of Riesz spaces . . .

Kunio Yamagata, The exchange property and direct sums of indecomposable injective modules. 